CASE REPORT

\title{
Upper airway obstruction masquerading as exercise induced bronchospasm in an elite road cyclist
}

\section{$K$ E Fallon}

Br J Sports Med 2004;38:e9 (http://www.bjsportmed.com/cgi/content/full/38/4/e9). doi: 10.1136/bjsm.2003.006486

This case concerns an elite road cyclist who complained of occasional dyspnoea and inspiratory difficulty during intense exercise. Clinical examination was normal and the final diagnosis was vocal cord dysfunction, a paradoxical closure of the vocal cords during inspiration which is highly associated with inspiratory stridor at high rates of ventilation. Awareness by the sports physician of this not uncommon condition is important to avoid misdiagnosis.

E xercise induced bronchospasm is a frequent diagnosis in athletes. However, when symptoms are atypical, standard lung function or provocation tests are normal, or response to medication is poor, other causes of exercise induced dyspnoea should be sought. This case concerns an elite road cyclist who complained of occasional dyspnoea and inspiratory difficulty during intense exercise. The final diagnosis was vocal cord dysfunction (VCD) which, with laryngochalasia and other conditions leading to stridor, is an important differential diagnosis in apparent cases of exercise induced bronchospasm.

\section{CASE REPORT}

Our patient was a 28 year old national champion road cyclist who initially presented with a 4-5 year history of noisy breathing. This occurred only during periods of very intense exercise, such as when hill climbing during a race, and on three occasions it had limited her performance. One of these occasions occurred at altitude in France and the others perhaps in association with weather changes. One episode was associated with feelings of anxiety following a tyre puncture.

The noisy breathing was, in fact, stridor which was inspiratory in nature, came on rapidly, and resolved equally quickly with rest. There was also a history of intermittent post-exercise cough which was not associated with wheeze or dyspnoea and which was referred to as "pursuiter's cough".

There was no history of breathlessness or wheeze in circumstances unrelated to exertion and there was no history of respiratory disease in childhood. Ingestion of alcohol was noted to cause nasal congestion and blockage and noisy nasal breathing, but it was stated that this was different to the presenting symptom. There was a maternal history of asthma and one of the patient's brothers had allergic rhinitis, sinusitis, and probable asthma. There was no personal history of atopy.

Clinical examination was completely normal and, in particular, there was no abnormality of the upper airways and the chest was clear with no adventitious sounds and good air entry. Spirometry at rest was normal. Forced expiratory volume in $1 \mathrm{~s}\left(\mathrm{FEV}_{1}\right)$ was $4.46 \mathrm{l}$ and forced vital capacity (FVC) $5.71 \mathrm{l}$, these values being 129 and $144 \%$ of predicted, respectively. Flows at low lung volumes were also normal. An exercise test for exercise induced bronchospasm, involving $10 \mathrm{~min}$ exercise on a cycle ergometer to maximum levels of exercise, was normal and reproduction of symptoms did not occur during the test. Representative values for $\mathrm{FEV}_{1}$ and $\mathrm{FEV}_{\mathrm{l}} / \mathrm{FVC}$ were as follows: at rest $4.12 \mathrm{l}$ and $77 \%$, at 1 min post exercise 4.48 and $95 \%$, at $5 \operatorname{min~} 4.54$ and $87 \%$, and at 10 min $4.12 \mathrm{l}$ and $85 \%$.

There was no relief of symptoms with use of an inhaled $\beta-2$ agonist prior to exercise and a 1 month trial of inhaled corticosteroid. Two episodes of stridor occurred during the period of use of the $\beta-2$ agonist. Further investigation included a histamine challenge test which was normal.

Based on the history of inspiratory stridor, lack of response to medication, and normal provocation tests, a diagnosis of intermittent upper airways obstruction was made with the differential diagnosis including paradoxical adduction of the vocal chords and exercise induced laryngochalasia. Referral was made to an ear, nose, and throat surgeon for laryngoscopy and detailed upper airway examination. These examinations were normal, in particular, there were no excessive mucosal folds or redundant tissue in the supraglottic area. During hyperventilation there was slight vibration of the aryepiglottic folds but no collapse of the supraglottic structures on inspiration. Although the conditions of severe exercise could not be replicated during the examination, these findings effectively exclude the diagnosis of exercise induced laryngochalasia.

Although it could not be proven absolutely, the provisional diagnosis was felt to be paradoxical adduction of the vocal chords (VCD).

As this condition may be associated with flattening of the inspiratory portion of a flow volume loop, this test was performed. It was normal. Again the patient was not symptomatic during the test.

A course of speech therapy was suggested but at 12 month follow up this had not been undertaken. The athlete noted that the episodes of stridor had reduced in number following diagnosis and that her program of international competition mitigated against a formal program of therapy.

\section{DISCUSSION}

Exercise induced bronchospasm is common, the prevalence in Olympic level athletes approximating $10-15 \% .^{1}$ The diagnosis has recently been questioned in some elite athletes and others whose airway narrowing may be induced as a physiological response to airway dehydration. ${ }^{2}$ This may be the explanation for the condition known colloquially as "pursuiter's cough". The diagnosis should also be questioned when the clinical history is atypical, provocation tests are negative, and if response to medication is poor.

Abbreviations: $\mathrm{FEV}_{1}$, forced expiratory volume in $1 \mathrm{~s} ; \mathrm{FVC}$, forced vital capacity; VCD, vocal cord dysfunction 


\section{Take home message}

Awareness of vocal cord dysfunction is important to avoid misdiagnosis.

Upper airway disease is not infrequently misdiagnosed as exercise induced bronchospasm and accounts for some cases of pseudosteroid resistant asthma. ${ }^{3}$ Inspiratory stridor is an important differentiating feature and this symptom has recently been described in $5.1 \%$ of a group of 370 elite athletes who were undergoing evaluation for exercise induced bronchospasm. ${ }^{4}$ In athletes participating in outdoor sports the prevalence was $8.3 \%$. Of the 19 athletes with this symptom, 18 were female and 10 also had exercise induced bronchospasm. ${ }^{4}$ There are a large number of conditions that can lead to tracheal or laryngeal obstruction including local infection, subglottic stenosis, vocal cord polyps, vocal cord paralysis, extrinsic compression, laryngomalacia, tumours and vascular lesions, and exercise induced anaphylaxis. Those most frequently mentioned in the context of exercise related obstruction are exercise induced laryngochalasia and VCD, otherwise known as paradoxical adduction of the vocal cords.

Exercise induced laryngochalasia or exercise induced laryngeal prolapse is most often seen in adolescent female athletes and in a recent small series two of the three patients were described as "gold medal" athletes. ${ }^{5}$ The clinical presentation is identical to that of VCD in that patients present with stridor, dyspnoea, inspiratory distress, and a rapid development and devolution of symptoms. Both exercise and odours are potential precipitating factors and the only finding on physical examination is inspiratory stridor. The flow volume loop may reveal plateauing of the inspiratory section. Differentiation from VCD may be difficult but at laryngoscopy, collapse of the posterior aryepiglottic folds over the vocal cords, mucosal oedema, and redundant mucosal tissue may be seen. Therapy involves laser surgery with removal of redundant tissue. This condition was effectively excluded in our patient.

VCD is a paradoxical closure of the vocal cords during inspiration which is highly associated with inspiratory stridor at high rates of ventilation, ${ }^{4}$ as typically occurs during intense competition. Normally the cords abduct during inspiration and adduct slightly or not at all during expiration. In VCD the anterior section of the vocal cords adduct during inspiration forming a small triangular opening posteriorly. An indication of the prevalence of this condition can be found in a study of military subjects with exertional dyspnoea where direct visualisation of the vocal cords by flexible laryngoscopy was performed before and after exercise to determine the incidence of VCD: $15 \%$ were found to have adduction of the cords. ${ }^{6}$ Newman et al found that $56 \%$ of 95 laryngoscopically confirmed patients with VCD also had asthma. ${ }^{7}$

VCD typically occurs in young females and may be precipitated by exercise, odours, and infections. VCD may be associated with gastro-oesophageal reflux which may lead to acute inflammation of the upper airway. ${ }^{8}$ It has been suggested that it is associated with a number of psychological disorders including anxiety, ${ }^{9}$ depression, and perfectionism. Anxiety was a factor in one of the episodes noted by our patient. It has been postulated, in the only study to date on VCD in elite athletes, that an athlete may subconsciously convert performance anxiety into laryngeal closure, this providing a physiological mechanism for some cases of "choking" during sport. ${ }^{10}$ Typical presenting symptoms include stridor, dyspnoea, inspiratory difficulty, throat tight- ness, and in some cases, a feeling of choking and cough. The disorder is highly variable and difficult to reproduce. The rapidity of onset and resolution of symptoms in relation to exercise is an important point in differentiation of VCD from asthma. Clinical examination is usually normal at rest and inspiratory stridor is the only physical sign present during an attack. Auscultation of the larynx during an episode, which allows for localisation of the source of the inspiratory sound, is highly useful in diagnosis. Whereas flattening of the inspiratory section of the flow volume loop is often cited as a typical finding in this condition, only $20 \%$ of subjects in the military study previously mentioned demonstrated this finding. ${ }^{6}$ Laryngoscopy may reveal adduction of the vocal cords but it is difficult to perform this procedure at the high levels of ventilation which may be required for precipitation of the condition.

Speech and relaxation therapy are the recommended treatment options. Only one study has assessed treatment for VCD in athletes. ${ }^{11}$ In this study 20 adolescent female athletes underwent speech therapy intervention which involved coordination of thoracic and abdominal respiratory movement patterns, elimination of paradoxical respiratory movements, and emphasis of the abdominal contribution to respiration during exercise. Some $95 \%$ of the participants reported the ability to control symptoms of VCD during exercise in the 6 month period following the intervention, while $80 \%$ no longer required asthma medication.

Significant improvements in both wheezing and anxiety have also been found to occur in cases of VCD following inhalation of heliox (20-40\% oxygen in helium) and it has been suggested that a heliox inhaler may be used in cases of VCD in a similar fashion to a $\beta$ agonist in asthma. ${ }^{12}$ More severe cases have been treated by injection of botulinum toxin type A into the vocal cords. ${ }^{13}$

\section{CONCLUSION}

VCD mimics and occurs in association with exercise induced bronchospasm. Differentiation may be made by careful history taking and is supported by normal standard lung function and bronchial provocation tests and lack of response to asthma medication. Laryngoscopy may assist in making a definitive diagnosis and in exclusion of other causes of upper airway obstruction. Awareness of this not uncommon condition is important for the sports physician as failure to recognise VCD may lead to multiple unnecessary investigations, hospital admission, prolonged inappropriate medication use, and failure to institute potentially beneficial treatment.

Conflict of interest: none declared.

Correspondence to: Kieran Edward Fallon, Australian Institute of Sport, Sports Medicine, PO Box 176, Belconnen, ACT 2616, Australia; fallonk@ausport.gov.au

Received 8 July 2003

Accepted for publication 15 July 2003

\section{REFERENCES}

1 Storms WW. Exercise induced asthma: diagnosis and treatment for the recreational and elite athlete. Med Sci Sports Exerc 1999:31:S33-8.

2 Anderson SD, Holzer K. Exercise-induced asthma: is it the right diagnosis in elite athletes? J Allergy Clin Immunol 2000;106:419-28.

3 Thomas PS, Geddes DM, Barnes PJ. Pseudo-steroid resistant asthma. Thorax 1999:54:352-65

4 Rundell KW, Spiering BA. Inspiratory stridor in elite athletes. Chest 2003; 123:468-74.

5 Bjornsdottir US, Gudmundsson K, Hiartarson H, et al. Exercise induced laryngochalasia: an imitator of exercise-induced bronchospasm. Ann Allergy Asthma Immunol 2000;83:387-91.

6 Morris MJ, Deal LE, Bean DR, et al. Vocal cord dysfunction in patients with exertional dyspnoea. Chest 1999;116:1676-82.

7 Newman KB, Mason UG III, Schmaling KB. Clinical features of vocal cord dysfunction. Am J Respir Crit Care Med 1995; 152:1382-6. 
8 Nielson DW, Heldt GP, Tooley WH. Stridor and gastrooesophageal reflux in infants. Paediatrics 1900;85:1034-9.

9 Brugman SM, Simons SM. Vocal cord dysfunction: don't mistake it for asthma. Physician Sportsmed 1998;26:63-74.

10 Mc Fadden ER III, Zawadski DK. Vocal cord dysfunction masquerading as exercise induced asthma: a physiologic cause for "choking" during athletic activities. Am J Respir Crit Care Med 1996;153:942-7.
11 Sullivan MD, Heywood BM, Beukelman DR. A treatment for vocal cord dysfunct ion in female athletes: an outcome study. Laryngoscope 2001;111:1751-5.

12 Weir M. Vocal cord dysfunction mimics asthma and may respond to heliox. Clin Pediatr 2002;41:37-41.

13 Maillard I, Schweizer V, Broccard A, et al. Use of botulinum toxin type A to avoid tracheal intubation or tracheostomy in severe paradoxical vocal cord movement. Chest 2000;118:874-6. 